\title{
Proton mobility in metallic copper hydride from high-pressure nuclear magnetic resonance
}

\author{
Thomas Meier $\odot,{ }^{1,}{ }^{*}$ Florian Trybel $\odot,{ }^{1}$ Giacomo Criniti,,${ }^{1}$ Dominique Laniel $\odot,{ }^{2}$ Saiana Khandarkhaeva $\odot,{ }^{1}$ Egor Koemets, ${ }^{1}$ \\ Timofey Fedotenko, ${ }^{2}$ Konstantin Glazyrin, ${ }^{3}$ Michael Hanfland $\odot,{ }^{4}$ Maxim Bykov $\odot{ }^{5}$ Gerd Steinle-Neumann, ${ }^{1}$ \\ Natalia Dubrovinskaia $\oplus^{2,6}$ and Leonid Dubrovinsky ${ }^{1}$ \\ ${ }^{1}$ Bayerisches Geoinstitut, Universität Bayreuth, 95440 Bayreuth, Germany \\ ${ }^{2}$ Material Physics and Technology at Extreme Conditions, Laboratory of Crystallography, Universität Bayreuth, 95440 Bayreuth, Germany \\ ${ }^{3}$ Deutsches Elektronen-Synchrotron (DESY), 22603 Hamburg, Germany \\ ${ }^{4}$ European Synchrotron Radiation Facility (ESRF), 38043 Grenoble Cedex, France \\ ${ }^{5}$ Department of Mathematics, Howard University, 2400 Sixth Street NW, Washington, DC 20059, USA \\ ${ }^{6}$ Department of Physics, Chemistry and Biology (IFM), Linköping University, SE-581 83 Linköping, Sweden
}

(Received 9 April 2020; revised 21 August 2020; accepted 15 September 2020; published 8 October 2020)

\begin{abstract}
The atomic and electronic structures of $\mathrm{Cu}_{2} \mathrm{H}$ and $\mathrm{CuH}$ have been investigated by high-pressure nuclear magnetic resonance spectroscopy up to $96 \mathrm{GPa}$, X-ray diffraction up to $160 \mathrm{GPa}$, and density functional theory-based calculations. Metallic $\mathrm{Cu}_{2} \mathrm{H}$ was synthesized at a pressure of $40 \mathrm{GPa}$, and semimetallic $\mathrm{CuH}$ at $90 \mathrm{GPa}$, found stable up to $160 \mathrm{GPa}$. For $\mathrm{Cu}_{2} \mathrm{H}$, experiments and computations show an anomalous increase in the electronic density of state at the Fermi level for the hydrogen $1 s$ states and the formation of a hydrogen network in the pressure range 43-58 GPa, together with high ${ }^{1} \mathrm{H}$ mobility of $\sim 10^{-7} \mathrm{~cm}^{2} / \mathrm{s}$. A comparison of these observations with results on $\mathrm{FeH}$ suggests that they could be common features in metal hydrides.
\end{abstract}

DOI: 10.1103/PhysRevB.102.165109

\section{INTRODUCTION}

Discovered in 1844 [1], metal hydrides are now of great interest in light of their potential as hydrogen storage and high-temperature superconducting materials [2-5]. Characterizing their stability, composition, electronic and transport properties provides crucial insight for a variety of applications, but metal hydrides continue to be enigmatic due to the highly variable metal-hydrogen bond. Following hydrogen uptake at elevated pressure $(P)$ (and temperature $T$ ), the electronic structure of metal hydrides can change significantly, giving rise to their semiconducting [6], metallic [7] to even superconducting behavior [3].

Hexagonal copper hydride (with wurtzite-type structure) was one of the first metal hydrides discovered in the 19th century [1]. As a highly reactive compound, it readily decomposes to the elements [8]. Over the past two decades, a number of high- $P$ copper hydrides were synthesized in the diamond anvil cell (DAC):

(i) Using dehydrogenated $\mathrm{Cu}$ and $\mathrm{H}_{2}$, cubic $\mathrm{CuH}_{0.4}$ with an $f c c$ arrangement of $\mathrm{Cu}$ atoms was formed at $P>14 \mathrm{GPa}$ [9]. By contrast, in the same work no reaction was observed when $\mathrm{Cu}$ foil was used as a starting material, suggesting that $\mathrm{CuH}_{0.4}$ does not form at equilibrium, rather, Burtovyy and Tkacz [9] inferred that the presence of small crystallites from the dehydrogenated copper hydride seems required for nucleation.

(ii) At $P>18 \mathrm{GPa}$ and room $T$, trigonal $\mathrm{Cu}_{2} \mathrm{H}$ with the anti-CdI $\mathrm{C}_{2}$ structure was synthesized and found stable up to $50 \mathrm{GPa}$ [10]. Donnerer et al. [10] also observed an $f c c$-based

*thomas.meier@uni-bayreuth.de copper hydride after decompression of trigonal $\mathrm{Cu}_{2} \mathrm{H}$ and recompression to $12.5 \mathrm{GPa}$, with a reported composition of $\mathrm{CuH}_{0.15}$, a significantly lower degree of hydrogenation.

(iii) Laser heating of pure copper grains with excess hydrogen resulted in the formation of $\mathrm{Cu}_{2} \mathrm{H}$ at $\sim 30 \mathrm{GPa}$, and $\mathrm{CuH}_{0.65}$ at $\sim 50 \mathrm{GPa}$ [11] (cubic with an $f c c$ arrangement of $\mathrm{Cu}$ atoms), the group 11 metal hydride with the highest documented hydrogen content at high $P$ found to this day.

Experimental probes in the DAC, the most important device used to generate high $P$ in solid state research of novel hydrides, have not been able to directly investigate electronic and dynamic properties of the hydrogen sublattice until recently. By combining magnetic flux tailoring techniques [12-14] and a modified DAC design [15], the accessible $P$ range for ${ }^{1} \mathrm{H}$ nuclear magnetic resonance (NMR) spectroscopy in the DAC was recently extended from $P<10 \mathrm{GPa}$ $[15,17]$ to beyond $100 \mathrm{GPa}[18,19]$.

A $P$-induced enhancement of $\mathrm{H}-\mathrm{H}$ interaction in iron monohydride $(\mathrm{FeH})$ - documented by an increased electron density of states at the Fermi level for the hydrogen $1 s$ states $\left(N_{\mathrm{H}-1 s}\left(E_{\mathrm{F}}\right)\right)$ - was described recently by our group [18] employing ${ }^{1} \mathrm{H}$-NMR spectroscopy in combination with electronic structure calculations, which was accompanied by the formation of a sublattice of increased electron localization connecting the hydrogen positions. However, similar studies have not yet been conducted for comparable metal hydrides to test the generality of such features. Here, we present results on copper hydrides using a combination of powder and single crystal synchrotron X-ray diffraction (XRD), in situ high- $P{ }^{1} \mathrm{H}-\mathrm{NMR}$ spectroscopy, and density functional theory (DFT) based computations, which enabled us to establish their structural and electronic properties, and 
determine ${ }^{1} \mathrm{H}$ mobility, and quantitatively compare to results for $\mathrm{FeH}$ [18].

\section{METHODS}

In this work, $\mathrm{CuH}_{x}$ compounds were synthesized by direct reaction of pure copper and paraffin in the laser-heated DAC $[18,20]$. Gaskets were manufactured by preindenting a $250 \mu \mathrm{m}$-thick rhenium foil to $\sim 25$ and $\sim 15 \mu \mathrm{m}$, respectively, and the hole for the sample chamber was laser drilled in the center of the flat indentation. All cells were loaded with paraffin oil (Sigma Aldrich Ltd.), serving as hydrogen reservoir, NMR reference signal, and $P$-transmitting medium. High-quality copper powder ( $5 \mathrm{~N}$ purity) was added with an estimated volume ratio of 1:10 to $1: 15$, in order to ensure hydrogen excess. The reaction of copper with paraffin was conducted at varying $P$ by first compressing the DAC and then using double-sided laser heating in the continuous wave mode with a nominal laser power of about $25-40 \mathrm{~W}$ to create $T$ in excess of $2000 \mathrm{~K}$.

\section{A. X-ray diffraction}

Two DACs with culets of 250 and $120 \mu \mathrm{m}$ in diameter were used for XRD experiments. Reaction of paraffin oil during laser heating with copper was confirmed by a change in optical properties of the sample and by the appearance of the Raman peak of microdiamonds formed from the carbon in the paraffin oil. Pressure inside the DAC was determined using the equations-of-state of unreacted copper, the rhenium gasket material, and the microdiamonds; $P$ estimated from the Raman shift of microdiamonds [21] was consistent with that obtained from the diamond edge of the anvils used for $P$ determination in the NMR experiments. Temperature was determined by multi-wave-length spectroradiometry.

High-resolution XRD measurements were performed at beamlines ID15 of the European Synchrotron Radiation Facility (ESRF) in Grenoble, France, and P02.2 of Petra III (DESY) in Hamburg, Germany. At beamline ID15, an X-ray beam of $30.142 \mathrm{keV}(0.41134 \AA)$ and a large-area MAR555 flat panel detector were used. At beamline P02.2, an X-ray beam of $42.87 \mathrm{keV}(0.2982 \AA)$ and a Perkin Elmer XRD1621 flat panel detector were used.

Phase identification was carried out based on powder diffraction patterns integrated from still and wide-scan images, using the DIOPTAS software [22]. Cell parameters were determined from LeBail refinement of selected powder diffraction patterns using JANA2006 [23]. A background correction was applied manually or by a Chebyshev polynomial, and peaks were fitted with pseudo-Voigt functions.

Single-crystal X-ray diffraction data were collected under cell rotation between $-35^{\circ}$ and $35^{\circ}$ to improve constraints on crystal symmetry. The data were analyzed using CrysAlisPro, including integration of intensities, frame scaling and empirical absorption corrections. Structural solutions and refinements were performed using the ShelX package $[24,25]$ in the WINGX software [16].

\section{B. Nuclear magnetic resonance measurements}

Four DACs for NMR measurements were prepared following the procedure described previously [15]. Diamond anvils with pairs of $250 \mu \mathrm{m}$ and $100 \mu \mathrm{m}$ culets were covered with a $1 \mu \mathrm{m}$ layer of copper using physical vapor deposition. By focused ion beam milling, Lenz lens structures were cut from the copper layer to form a double- and triple-stage NMR resonator setup for the 250 and $100 \mu \mathrm{m}$ anvils, respectively. To insure insulation between the resonators and the metallic gasket, the latter was covered by $500 \mathrm{~nm}$ of $\mathrm{Al}_{2} \mathrm{O}_{3}$ using chemical vapor deposition. Helmholtz excitation coils were manufactured from $80 \mu \mathrm{m}$-thick PTFE (Teflon) insulated copper wire consisting of 4 to 5 turns with an inner diameter of $\sim 3 \mathrm{~mm}$. Both coils were fixed onto the diamond backing plates such that the copper coated anvils are at the coils' center. After cell loading and closure, the excitation coils were connected to form a Helmholtz coil-like arrangement.

The NMR cells were inserted into a standard in-house built NMR probe for use with a magnetic field of $\sim 1030 \mathrm{mT}$ generated by an electron spin resonance electromagnet, corresponding to a resonance frequency $\left(\omega_{0}\right)$ of the hydrogen nuclei of $43.851 \mathrm{MHz}$. In order to limit radio frequency excitation to a region of interest of $\sim 2000 \mathrm{ppm}(90 \mathrm{kHz}$ at $1030 \mathrm{mT}$ ), we recorded high- $P{ }^{1} \mathrm{H}-\mathrm{NMR}$ spectra using amplitude-modulated band-selective pulses with uniform response and pure phase (E-BURP) [26]. Relaxation times $\left(T_{1}\right)$ were obtained using an I-BURP/E-BURP inversion recovery sequence. ${ }^{1} \mathrm{H}$-NMR spectra were found broadened (see Supplemental Material [27]; Fig. S1) by pronounced Knight shift anisotropies $(\Delta K)$.

In order to extract the isotropic values of the Knight shift $\left(K_{\mathrm{H}}\right)$, we developed a Python script for line-shape simulations adopted to the special experimental needs of high- $P$ NMR. Simulation of the NMR line shapes (exemplary shown in Fig. S2 in the Supplemental Material [27]) was carried out following [28-30]. We used the standard expression for the resonance frequency distribution $\omega$ for a given $\Delta K$ and $K_{H}$,

$\omega(\alpha, \beta)=\omega_{0} K_{\mathrm{H}}+\Delta K \cdot\left(\frac{3 \cos ^{2} \beta-1}{2}+\frac{\eta}{2} \sin ^{2} \beta \cos (2 \alpha)\right)$,

where the Euler angles $\alpha$ and $\beta$ describe the orientation of the crystallites with respect to the external magnetic field $B_{0}$.

We used the Haeberlein convention for the NMR shift tensor [31],

$$
\begin{gathered}
\Delta K=K_{z z}-\frac{K_{\mathrm{xx}}+K_{\mathrm{yy}}}{2}, \\
K_{\mathrm{H}}=\frac{K_{\mathrm{xx}}+K_{\mathrm{yy}}+K_{\mathrm{zz}}}{3}, \\
\eta=\frac{K_{y y}-K_{x x}}{K_{z z}} \quad \text { with }\left|K_{z z}\right|>\left|K_{x x}\right|>\left|K_{y y}\right| .
\end{gathered}
$$

The asymmetry parameter $\eta$ accounts for the deviation from sphericity of the Knight shift tensor $K$.

The line-shape function used to fit the spectra is

$$
P(\omega)=\int_{-1}^{1} \frac{\mu}{4 \pi} \sin (\beta(\omega, \alpha))\left|\frac{\partial \beta(\omega, \alpha)}{\partial \omega}\right| d(\cos (2 \alpha)),
$$

where $\beta(\omega, \alpha)$ is the inverse function of Eq. (1) with respect to $\beta$, and $\mu$ accounts for the multiplicity of spectral functions. In order to obtain $K_{\mathrm{H}}$, we calculated $P(\omega)$ for a given $\omega_{0}$, and 
(a)

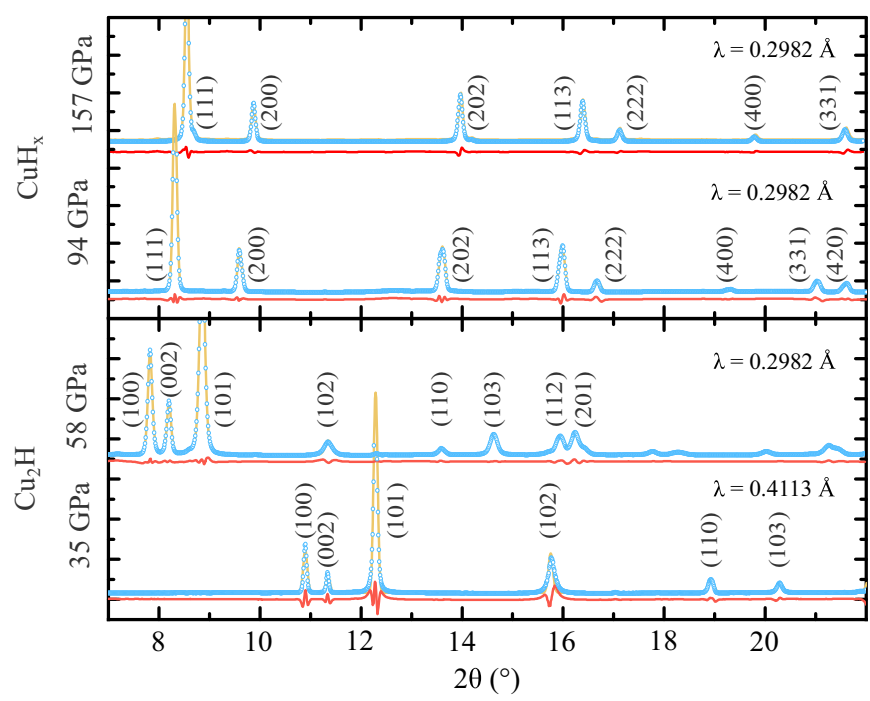

(b)

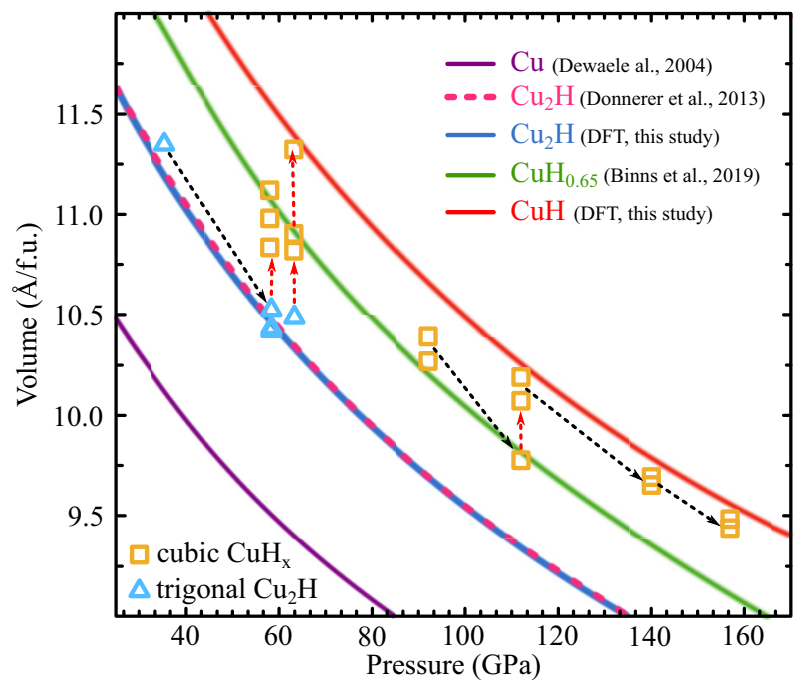

FIG. 1. (a) Examples of powder X-ray diffraction patterns of $\mathrm{Cu}_{2} \mathrm{H}$ and $\mathrm{CuH}$ synthesized at different pressures; yellow lines are Rietveld fits to the experimental data (blue dots); red lines denote the residuals. Indexed peaks are for the $P \overline{3} m 1 \mathrm{Cu}_{2} \mathrm{H}$ and $F m \overline{3} m \mathrm{CuH}_{x}$ structures. (b) Equations-of-state of the copper hydride phases and pure $\mathrm{Cu}$ : the equations-of-state for pure copper [41] and $\mathrm{CuH}_{0.65}$ [11] are shown in purple and green, respectively. The pink dashed line denotes the equation-of-state for $P \overline{3} m 1 \mathrm{Cu}_{2} \mathrm{H}$ [10]. The results of a third-order Birch-Murnaghan fit to the DFT ground-state energies are shown by a blue curve for $\mathrm{Cu}_{2} \mathrm{H}$ and in red for $\mathrm{CuH}$. The equation-of-state parameters can be found in Table S1 in the Supplemental Material [27]. Open orange squares $\left(\mathrm{CuH}_{x}\right)$ and blue triangles $\left(\mathrm{Cu}_{2} \mathrm{H}\right)$ represent diffraction data, and the arrows illustrate the experimental pathways of heating (red) and compression (black).

optimized with respect to the experimental data by varying $\Delta K, K_{\mathrm{H}}$, and $\eta$ (Fig. S3 in the Supplemental Material [27]).

\section{Density functional theory calculations}

All electronic structure calculations were performed with QUANTUM ESPRESSO [32,33] with the projector augmented wave (PAW) approach [34]. We used the generalized gradient approximation by Perdew-Burke-Enzerhofer (PBE) [35] to exchange and correlation with the corresponding PAW-PBE potential files. For both $\mathrm{Fe}$ and $\mathrm{Cu}$, a valence electron configuration that includes electronic states $3 s$ and higher is applied, appropriate to extreme $P$ [36]. Convergence tests for static electronic structure simulations led to a cutoff energy for the plane-wave expansion of $170 \mathrm{Ry}(\mathrm{Cu})$ and $120 \mathrm{Ry}(\mathrm{Fe})$ and to reciprocal space sampling with a Monkhorst-Pack [37] grid of $32 \times 32 \times 32$. In addition to the total electronic densityof-states (DOS), site and orbital projections were computed (Fig. S4 in the Supplemental Material [27]), with particular attention to the crystal field splitting of the $3 d$ electronic states. Equations-of-state of $\mathrm{CuH}$ and $\mathrm{CuH}_{2}$ were fitted based on the ground state energies for various unit cell volumes $(V)$ using a Birch-Murnaghan formulation [38]. Fit parameters are summarized in Table S1 of the Supplemental Material [27].

Molecular dynamics (MD) simulations were performed for cells of 54 and 96 atoms for $\mathrm{Cu}_{2} \mathrm{H}$ and $\mathrm{FeH}$, respectively, with a Monkhorst-Pack [37] grid of $2 \times 2 \times 2$. Calculations were performed in the canonical ensemble, and $T$ was controlled by an Anderson thermostat [39]. We employed a timestep of 0.1 fs to account for the fast hydrogen dynamics [40] and ran the simulations for a duration of $1.6 \mathrm{ps}$. Diffusion coefficients were calculated from the mean square displacements obtained via MD trajectories (see Supplemental Material; Fig. S5 [27]).

\section{RESULTS AND DISCUSSION}

\section{A. Structure and composition of $\mathrm{Cu}$-hydrides}

After laser heating at $P=35 \mathrm{GPa}$, a trigonal $\mathrm{Cu}_{2} \mathrm{H}$ compound (space group $P \overline{3} \mathrm{~m} 1$ ) formed, as determined by powder (Fig. 1(a), Table S2 in the Supplemental Material [27]) and single crystal diffraction (Tables S3 and S4 in the Supplemental Material [27]). The trigonal symmetry of $\mathrm{Cu}_{2} \mathrm{H}$ is based on a distorted hexagonal structure, with $h c p$-forbidden peaks present (e.g., 001, 003). This phase remained stable under compression to at least $P=90 \mathrm{GPa}$ at room $T$. At $P=35$ and $58 \mathrm{GPa}$, its atomic volumes (per $\mathrm{Cu}$ atom, $V / \mathrm{Cu}$ ) are 11.27(2) and 10.44(1) $\AA^{3}$, in agreement with values expected from the equations-of-state previously reported from experiments [10] and computed here (Fig. 1(b)).

Laser heating of $\mathrm{Cu}_{2} \mathrm{H}$ or pristine copper with paraffin at $P>50 \mathrm{GPa}$ resulted, in agreement with previous observations [11], in the formation of a cubic phase with an $f c c$ arrangement of $\mathrm{Cu}$ atoms. The same phase has been found stable upon laser heating of the sample up to $P \approx 160 \mathrm{GPa}$, the highest $P$ reached in this study. The sample's $V / \mathrm{Cu}$ at $P=58,94$, and $112 \mathrm{GPa}\left(11.11(2), 10.27(4), 9.80(6) \AA^{3}\right.$ ) agree with those derived from the equation-of-state of $f c c$-structured $\mathrm{CuH}_{0.65}$ [11] (Fig. 1(b)).

After repeated heating and $T$ quench of the cubic phase at $P$ $\geqslant 110 \mathrm{GPa}$, an increase of $\mathrm{V} / \mathrm{Cu}$ was observed (Fig. 1(b)) and values eventually became larger than expected for previously reported copper hydrides: at $P=157 \mathrm{GPa}$, for example, $V / \mathrm{Cu}$ 


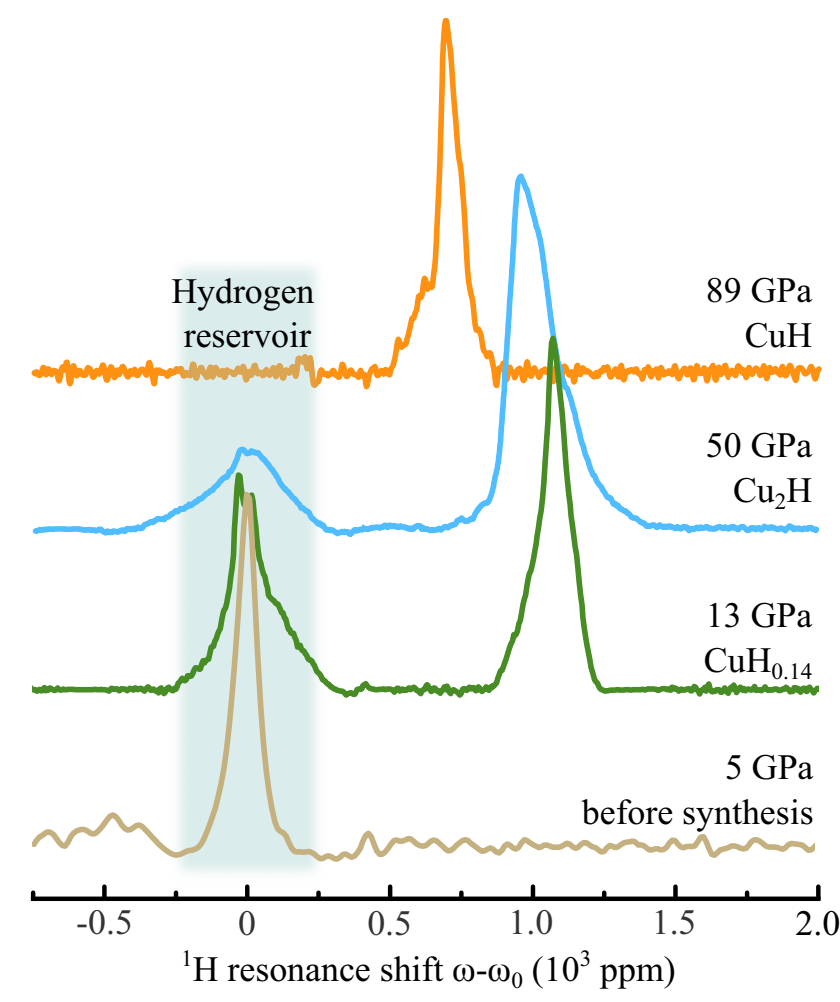

FIG. 2. Representative ${ }^{1} \mathrm{H}-\mathrm{NMR}$ spectra of copper hydrides, $\mathrm{CuH}, \mathrm{Cu}_{2} \mathrm{H}$, and $\mathrm{CuH}_{0.15}$, formed after laser heating of copperparaffin loaded cells at 89 and $50 \mathrm{GPa}$, and on decompression at $13 \mathrm{GPa}$.

reaches 9.48(2) $\AA^{3}$ vs $9.03 \AA^{3}$ for $\mathrm{CuH}_{0.65}$ [11] and $7.79 \AA^{3}$ for $\mathrm{Cu}$ [41] based on the reported equations-of-state. Thus, our data suggest that at $P \geqslant 110 \mathrm{GPa}$, we synthesized a copper hydride with a larger hydrogen content than previously reported at high $P[10,11]$.

An exact determination of the hydrogen content of $\mathrm{Cu}$ compounds formed in different experiments is difficult, but we note that the DFT-based results reproduce the experimental equation-of-state for trigonal $\mathrm{Cu}_{2} \mathrm{H}$ well (Fig. 1(b)). The experimental data compares similarly well with the DFT-based calculations on the equation-of-state for $\mathrm{NaCl}$-structured $\mathrm{CuH}$ (Fig. 1(b)), and we therefore infer that the cubic copper hydride we synthesized at high $P$ under $T$ cycling has a ratio of $\mathrm{Cu} / \mathrm{H}=1(\mathrm{CuH})$, (Table S1 in the Supplemental Material [27]). Laser heating at $P=140 \mathrm{GPa}$ produced no further increase in $V$ for $\mathrm{CuH}$, implying that either the $\mathrm{H}$ reservoir was exhausted or that the $\mathrm{Cu}$ hydride reached its maximum $\mathrm{H}$ storage capacity.

\section{B. NMR data and interpretation}

The chemical compositions of copper hydrides and their respective stability fields provide the basis for the discussion and interpretation of ${ }^{1} \mathrm{H}$-NMR data. Figure 2 shows representative ${ }^{1} \mathrm{H}$-NMR spectra of the copper hydrides at different pressures (cf. Fig. S1 in the Supplemental Material for all ${ }^{1} \mathrm{H}-\mathrm{NMR}$ spectra [27]). The NMR signal from the copper-paraffin sample $(P=5 \mathrm{GPa}$ before heating) originates solely from the paraffin reservoir, and both the full width at half maximum
(FWHM) of the NMR signal and the relaxation rate agree well with previous studies [14,18]. As $\omega_{0}$ of the paraffin reservoir does not change considerably with $P$ (Fig. 2), it was used as a reference. Additional signals appeared after laser heating at $P=50$ and $89 \mathrm{GPa}$, assigned to $\mathrm{Cu}_{2} \mathrm{H}$ and $\mathrm{CuH}$, respectively. During decompression of $\mathrm{Cu}_{2} \mathrm{H}$ to $P \leqslant 16 \mathrm{GPa}$, the spectrum was interpreted as that of $\mathrm{CuH}_{0.15}$, given the agreement in terms of transition $P$ with previous experimental work [10,42]. The successive decrease of the resonance shift with increasing amount of hydrogen is in agreement with other metal hydrides synthesized at ambient $P$ [43-47].

Figure 3(a) shows the relative changes of $K_{\mathrm{H}}$ as a function of compression $V / V_{0}$ for the three copper hydrides synthesized, $\mathrm{CuH}, \mathrm{Cu}_{2} \mathrm{H}$, and $\mathrm{CuH}_{0.15}$ [for $K_{\mathrm{H}}$ as a function of the hydrogen-hydrogen distances $r_{\mathrm{HH}}$, see Fig. S3(a) in the Supplemental Material [27]], as well as for cubic $\mathrm{FeH}$ from our previous work [18]. $\mathrm{Cu}_{2} \mathrm{H}$ and $\mathrm{FeH}$ follow a slope expected for a free electron gas-like system $\left(K_{\mathrm{H}} \propto V^{2 / 3}[18]\right)$, while both $\mathrm{CuH}_{0.15}$ and $\mathrm{CuH}$ violate Fermi gas ideality, indicating that these phases might at best be considered as bad metals or semimetals, in agreement with recent DFT calculations [48].

The $V$ dependence of $K_{\mathrm{H}}$ for $\mathrm{Cu}_{2} \mathrm{H}$ displays a deviation from free electron gas behavior for $43 \mathrm{GPa} \lesssim P \lesssim 58 \mathrm{GPa}$, similar to $\mathrm{FeH}$ for $64 \mathrm{GPa} \lesssim P \lesssim 110 \mathrm{GPa}$ [18]. This effect can also be seen in the dependence of the electronic DOS at the Fermi energy $N\left(E_{\mathrm{F}}\right)$ from the DFT simulations, consisting of the sum of the $4 s$ electron DOS of $\mathrm{Cu}$ and the $1 s$ electron DOS of $\mathrm{H}$ (Fig. 3(a)). Interestingly, the contribution of the hydrogen $1 s$ electron to $N\left(E_{\mathrm{F}}\right)$ for $\mathrm{Cu}_{2} \mathrm{H}$ and $\mathrm{FeH}$ (Fig. 4(a)) gradually increases with decreasing distance between hydrogens $\left(r_{\mathrm{HH}}\right)$ (Fig. S4 in the Supplemental Material [27]), similar to the total DOS (Fig. 3(a)). This observation suggests that conduction electron density from the uncompensated $4 s$ states in $\mathrm{Cu}_{2} \mathrm{H}$ or the uncompensated $3 d-t_{2 \mathrm{~g}}$ states in $\mathrm{FeH}[18]$ is transferred to $N_{\mathrm{H}-1 s}\left(E_{\mathrm{F}}\right)$, respectively. By contrast, $N_{\mathrm{H}-1 s}\left(E_{\mathrm{F}}\right)$ of semimetallic $\mathrm{CuH}$ shows a strongly negative slope (Fig. 4(a)). Given the striking similarity of the observed behavior in $K_{\mathrm{H}}$ and $N\left(E_{\mathrm{F}}\right)$ for $\mathrm{Cu}_{2} \mathrm{H}$ with that of $\mathrm{FeH}$ [18], we infer that they can be explained by the same $P$-induced formation of a sublattice of increased electron localization connecting the hydrogen positions accompanied by an increasing $N_{\mathrm{H}-1 s}\left(E_{\mathrm{F}}\right)$ in trigonal $\mathrm{Cu}_{2} \mathrm{H}$ at $\mathrm{P} \gtrsim 40 \mathrm{GPa}$.

Figure $3(\mathrm{~b})$ shows the dependence of the relative proton NMR resonance linewidths on $r_{\mathrm{HH}}$ in both metallic phases, $\mathrm{Cu}_{2} \mathrm{H}$ and $\mathrm{FeH}$, and in semimetallic $\mathrm{CuH}$. For $r_{\mathrm{HH}}>2.45 \AA$ $\left(P=43 \mathrm{GPa}\right.$ for $\mathrm{Cu}_{2} \mathrm{H}$ and $P=64 \mathrm{GPa}$ for $\left.\mathrm{FeH}\right)$, linewidths of the NMR signals for both transition metal hydrides and semimetallic $\mathrm{CuH}$ increase with $P$ due to dipolar coupling of hydrogen spin- $1 / 2$ nuclei, indicated by a $\Delta \omega \propto r_{\mathrm{HH}}^{-3}$ slope $[49,50]$. At higher $P$, i.e., $r_{\mathrm{HH}}<2.45 \AA$, the NMR absorption linewidths for $\mathrm{Cu}_{2} \mathrm{H}$ (Fig. S1 in the Supplemental Material [27]) and $\mathrm{FeH}$ decrease significantly. Values of $\Delta K$ and $\eta$ for $\mathrm{Cu}_{2} \mathrm{H}$ from spectral simulations were found to be almost pressure independent at $r_{\mathrm{HH}}>2.45 \AA$. For smaller $r_{\mathrm{HH}}$ (i.e., at higher $P$ ), $\Delta K$ decreases abruptly and $\eta \approx 0.0$ (Fig. S3 in the Supplemental Material [27]), and spectra approach a Voigtian line shape (Fig. S1 in the Supplemental Material [27]), which we attributed to the modification of electronic structure in $\mathrm{Cu}_{2} \mathrm{H}$ at similar $r_{\mathrm{HH}}$ as previously reported for $\mathrm{FeH}$ [18]. 
(a)

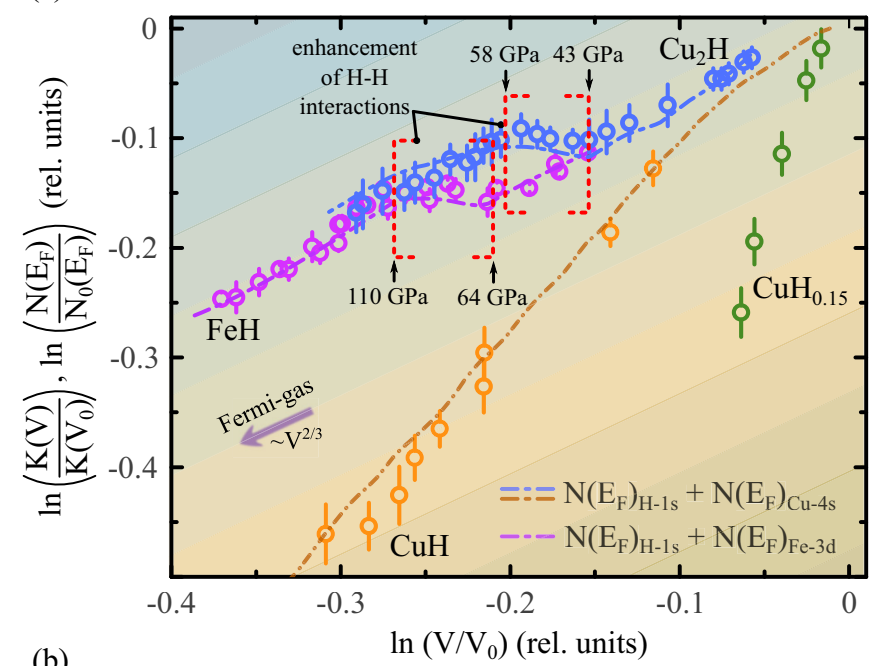

(b)

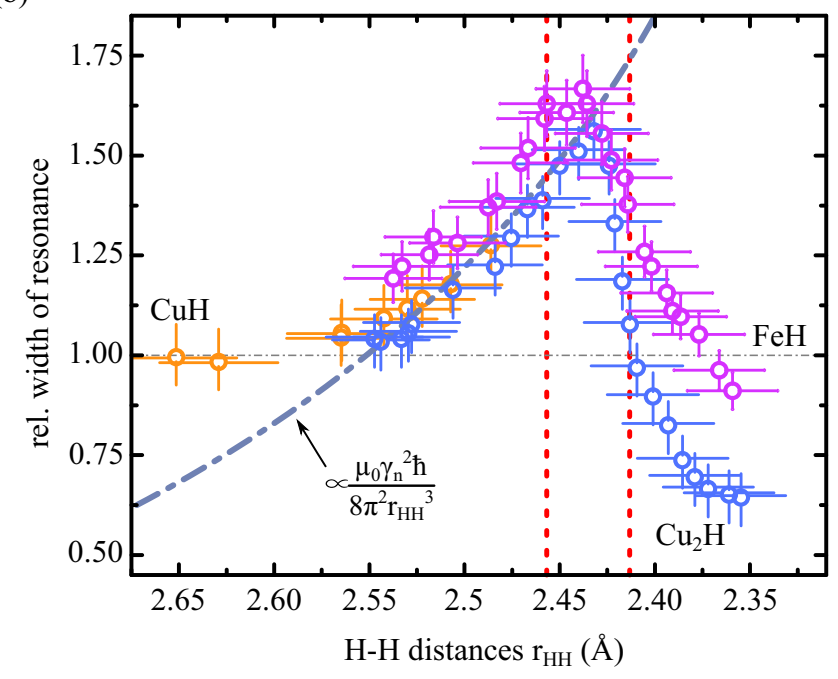

FIG. 3. Comparison of experimental NMR data and DFT-based electronic structure calculations. (a) Double logarithmic power plot of relative changes in the NMR Knight shift $K_{\mathrm{H}}(V)$ and the electron density-of-states at the Fermi energy $N\left(E_{\mathrm{F}}\right)$ as a function of compression. Experimental data (blue, magenta, orange, and green) are normalized to $K_{\mathrm{H}}\left(V_{0}\right)$ using $V_{0}$ from the respective equation-of-state from Tkacz et al. [42] $\left(\mathrm{CuH}_{0.15}\right)$ and DFT computations from this study $\left(\mathrm{CuH}\right.$ and $\mathrm{Cu}_{2} \mathrm{H}$ ) (Table $\mathrm{S} 1$ in the Supplemental Material [27]). The blue, magenta, and orange dashed lines (splines through computed values) show the respective volume dependence of $N\left(E_{\mathrm{F}}\right)$. Diagonal color stripes are guides to the eye, representing a $\propto V^{2 / 3}$ scaling for free-electron Fermi-gas-like behavior [18]. Black arrows denote respective pressure points. Data and results for $\mathrm{FeH}$ were taken from our previous work [18]. (b) Relative FWHM linewidths of ${ }^{1} \mathrm{H}-\mathrm{NMR}$ spectra as a function of the $\mathrm{H}-\mathrm{H}$ distance $r_{\mathrm{HH}}$ for $\mathrm{CuH}$, $\mathrm{Cu}_{2} \mathrm{H}$, and $\mathrm{FeH}$. The dotted blue line depicts the theoretical linewidth dependence for pure dipolar broadening and vertical red dashed lines show the $r_{\mathrm{HH}}$ corresponding to the pressure intervals marked by the arrows in (a).

The deviation of $K_{\mathrm{H}}$ from a Fermi-gas-like behavior can be estimated via the Korringa ratio $\left(K_{\mathrm{H}}^{2} T / R_{1}\right.$, with the relaxation rate $R_{1}=1 / T_{1}$ ), normalized with its free electron gas equivalent [51,52], $\frac{h}{8 \pi^{2} k_{\mathrm{B}} T}\left(\frac{\gamma_{\mathrm{e}}}{\gamma_{\mathrm{n}}}\right)^{2}$, where $k_{\mathrm{B}}$ is the Boltzmann (a)

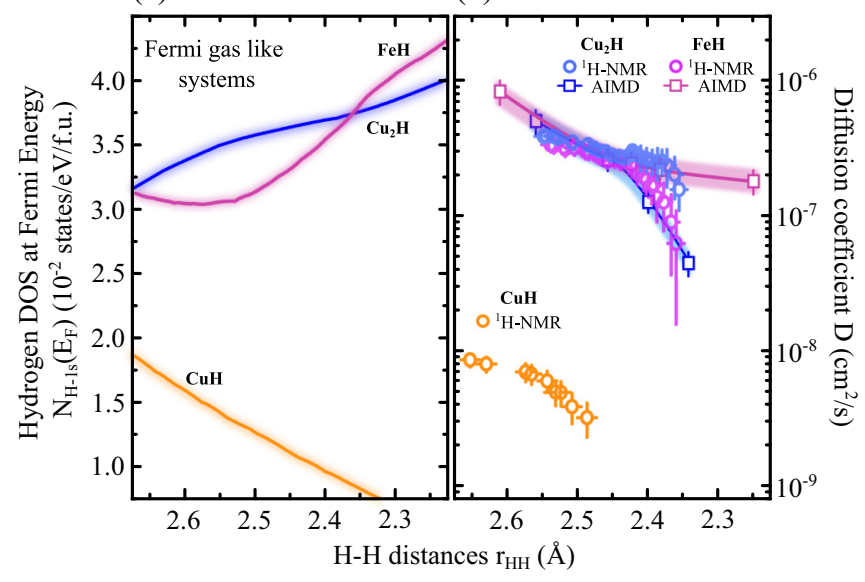

FIG. 4. Comparison of electronic structure and proton diffusivities. (a) Hydrogen 1-s electron density-of-state contribution $N_{\mathrm{H}-1 s}\left(E_{\mathrm{F}}\right)$ for $\mathrm{CuH}, \mathrm{Cu}_{2} \mathrm{H}$, and $\mathrm{FeH}$ as a function of the $\mathrm{H}-\mathrm{H}$ distance in the structures (pressure increasing to the right). (b) Proton diffusion coefficients extracted from NMR data (blue, purple, and orange circles) and computed via DFT-based molecular dynamics simulations (blue and magenta squares and lines) for metallic $\mathrm{Cu}_{2} \mathrm{H}$ and $\mathrm{FeH}$, and the semimetal $\mathrm{CuH}$. The values fall into two groups: (i) high $N_{\mathrm{H}-1 s}\left(E_{\mathrm{F}}\right)$ values and high diffusivity for the metallic compounds and (ii) small $N_{\mathrm{H}-1 s}\left(E_{\mathrm{F}}\right)$ values accompanied by a smaller diffusion coefficient in $\mathrm{CuH}$.

and $h$ is Planck's constant, $\gamma_{\mathrm{e}}$ and $\gamma_{\mathrm{n}}$ are the gyromagnetic ratios of the electron and the hydrogen nucleus, respectively. We find this ratio to be larger than one for $r_{\mathrm{HH}} \gtrsim 2.45 \AA$, indicating significant electron-electron correlation for both $\mathrm{FeH}$ and $\mathrm{Cu}_{2} \mathrm{H}$. For smaller $r_{\mathrm{HH}}$ the ratio approaches unity (Fig. S6 in the Supplemental Material [27]); this finding suggests that enhanced Fermi contact hyperfine interactions dominate the electronic structure at high $P$.

Since diffraction data and computational results on $\mathrm{Cu}_{2} \mathrm{H}$ (this study) and $\mathrm{FeH}[18,20]$ do not show signs of structural transitions within the experimental $P$ range, line narrowing effects cannot be related to atomic rearrangements. Rather, linewidth changes for two different transition metal hydrides at comparable $r_{\mathrm{HH}}$ suggest that electronic and dynamic effects on the hydrogen spin system must be considered.

\section{Proton diffusivities}

Hydrogen spin-lattice relaxation rates $R_{1}$ in metal hydrides originate from two mechanisms, representing electronic hyperfine interactions $R_{1}^{\mathrm{e}}$ and proton diffusion $R_{1}^{\mathrm{d}}$ [53]:

(i) $R_{1}^{\mathrm{e}}$ of hydrogen nuclei, from the interaction with conduction electron spins, can be calculated by a Korringa-like behavior for a Fermi gas $[51,52]$ with

$$
K_{\mathrm{H}}^{2}=\frac{h}{8 \pi^{2} k_{\mathrm{B}} T}\left(\frac{\gamma_{\mathrm{e}}}{\gamma_{\mathrm{n}}}\right)^{2} R_{1}^{\mathrm{e}},
$$

as described above. $K_{\mathrm{H}}$ originates from contact hyperfine interaction between the hydrogen $1 s$ electron orbital and the 
proton via

$$
K_{\mathrm{H}}=\frac{2 \mu_{0} \mu_{\mathrm{B}}}{A} H_{\mathrm{hf}}^{s} N\left(E_{\mathrm{F}}\right),
$$

with $\mu_{0}$ the permeability of free space, $A$ is Avogadro's number, $\mu_{\mathrm{B}}$ is the Bohr magneton, and $H_{\mathrm{hf}}^{\mathrm{s}}$ the hyperfine field [18].

(ii) A modulation of the relaxation rate, from the diffusive motion of hydrogen nuclei $\left(R_{1}^{\mathrm{d}}\right)$ in crystalline systems, can be expected to be well described by the theory of Bloembergen, Pound, and Purcell [54] by the use of a single correlation time for a stochastic undirected motion of hydrogen atoms as

$$
R_{1}^{\mathrm{d}}=\frac{3 \pi}{10} \frac{\gamma_{\mathrm{n}}^{4} h^{2} N_{0}}{a D},
$$

where $N_{0}$ is the number density of atoms, $D$ is the hydrogen diffusion coefficient, and $a$ is the distance of closest approach, which is at the order of $r_{\mathrm{HH}}$.

Calculating the relaxation rate $R_{1}$ (Fig. S6 in the Supplemental Material [27]) in the extreme narrowing limit [49], i.e., $R_{1}=R_{1}^{\mathrm{e}}+R_{1}^{\mathrm{d}}$, from the experimental linewidths via the relationship $R_{1} \approx \pi \cdot \Delta \omega$, and subtracting the conduction electron contribution $R_{1}^{\mathrm{e}}$ calculated via Eq. (6) gives access to $D$ of the protons in transition metal hydrides at high $P$, following early work at ambient $P[55,56]$. Figure 4(b) shows experimentally derived and computed $D$ as a function of $r_{\mathrm{HH}}$ for $\mathrm{Cu}_{2} \mathrm{H}, \mathrm{FeH}$, and semimetallic $\mathrm{CuH}$.

Values for $D$ decrease from $D_{\mathrm{Cu}_{2} \mathrm{H}} \sim 3.8(4) \times 10^{-7} \mathrm{~cm}^{2} / \mathrm{s}$ at $15 \mathrm{GPa}$ and $D_{\mathrm{FeH}} \sim 3.3(6) \times 10^{-7} \mathrm{~cm}^{2} / \mathrm{s}$ at $33 \mathrm{GPa}$ to $D_{\mathrm{Cu}_{2} \mathrm{H}} \sim 1.6(9) \times 10^{-7} \mathrm{~cm}^{2} / \mathrm{s}$ and $D_{\mathrm{FeH}} \sim 0.8(9) \times$ $10^{-7} \mathrm{~cm}^{2} / \mathrm{s}$ at 96 and $202 \mathrm{GPa}$, respectively. A steeper slope of $D$ for $r_{\mathrm{HH}}<2.42 \AA$ coincides with a decrease in spin-lattice relaxation time $T_{1}$ for $V / V_{0} \lesssim 0.8$, observed in both $\mathrm{Cu}_{2} \mathrm{H}$ (Fig. S3 in the Supplemental Material [27]) and $\mathrm{FeH}$. Proton diffusivity in $\mathrm{CuH}$ was found approximately two orders of magnitude smaller than that for $\mathrm{Cu}_{2} \mathrm{H}$. Hydrogen diffusion coefficients calculated from DFT MD simulations [Figs. 4(b) and S5 in the Supplemental Material [27]] agree with our ${ }^{1} \mathrm{H}$-NMR derived results. The correlation between an increased density-of-states at $E_{\mathrm{F}}$ and high values of $D$ in the metallic hydrides, $\mathrm{Cu}_{2} \mathrm{H}$ and $\mathrm{FeH}$, support a causal relation.

Proton mobility investigated in other metal hydride systems by means of standard as well as pulsed-field gradient NMR are typically found to be in the range $10^{-13}-10^{-7} \mathrm{~cm}^{2} / \mathrm{s}$ [53,55-59]. Diffusivities for $\mathrm{Cu}_{2} \mathrm{H}$ and $\mathrm{FeH}$ are at the upper limit of this broad range, suggesting that proton self-diffusion plays a more significant role in high- $P$ metal hydrides than in hydrides stabilized at ambient conditions. Given the fact that comparable metal hydrides, such as $\mathrm{TiH}_{1.66}$ [59] and $\mathrm{VH}_{0.39-0.85}$ [55], show significantly lower $D$ values for the protons in the range $10^{-13}-10^{-10} \mathrm{~cm}^{2} / \mathrm{s}$ at larger $r_{\mathrm{HH}}$, it stands to reason that proton self-diffusion in $\mathrm{H}$-rich polyhydrides, such as $\mathrm{LaH}_{10}, \mathrm{YH}_{10}$ or $\mathrm{CaH}_{6}$ with $r_{\mathrm{HH}}<1.2 \AA$ [60-62], approaches values several orders of magnitude larger than for $\mathrm{Cu}_{2} \mathrm{H}$ and $\mathrm{FeH}$. Recent computational results $[63,64]$ suggest that atomic quantum fluctuations and significant hydrogen zero-point energies strongly influence the high- $P$ stability of these H-rich polyhydrides. Thus, ab initio computations on diffusivity including nuclear quantum effects would shed further light on these phenomena.

\section{CONCLUSION}

In this work, three stable copper hydride phases of varying hydrogen content $\left(\mathrm{CuH}_{0.15}, \mathrm{Cu}_{2} \mathrm{H}\right.$, and $\left.\mathrm{CuH}\right)$ were synthesized at high pressure in the diamond anvil cell. For $\mathrm{Cu}_{2} \mathrm{H}$, we found an ideal metallic trend in the ${ }^{1} \mathrm{H}-\mathrm{NMR}$ Knight shift, with an unexpected change in slope in a pressure-range of $43-58 \mathrm{GPa}$, associated with an increase of conduction electron density-of-states at the Fermi energy of the hydrogens $1 s$ electron orbitals, similar to our earlier observations in metallic FeH [18]. For $\mathrm{FeH}$, the increase was observed at $P=64-110 \mathrm{GPa}$ and found to be correlated with the formation of a sublattice of increased electron density between the hydrogen atoms, intercalating the metal host lattice.

A line-shape and relaxation-rate analysis of the NMR spectra revealed, in agreement with molecular dynamics simulations, a significantly increased diffusivity of protons in the metallic hydride compounds $\left(\mathrm{Cu}_{2} \mathrm{H}\right.$ and $\left.\mathrm{FeH}\right)$, in contrast to the semimetallic $\mathrm{CuH}$ with proton diffusivity two orders of magnitude slower, accompanied by a significantly smaller electron density-of-states at the Fermi energy. Correlating these effects with the average $\mathrm{H}-\mathrm{H}$ distances, we find that in both metallic hydride compounds hydrogen-interactions significantly increase at almost identical $r_{\mathrm{HH}}(2.45 \AA)$, implying that the observed high diffusivities may only weakly depend on the host transition metal ions. This insight may provide an important step in the future search and design of novel hydride-based superconductors at high pressure for which a $\mathrm{H}$ sublattice of increased electron density was predicted to be a requirement for high-temperature superconductivity [60].

\section{ACKNOWLEDGMENTS}

We thank N. Miyajima for help with the FIB milling. The authors thank the German Research Foundation (Deutsche Forschungsgemeinschaft, DFG, Projects No. DU 954/11-1, No. DU 393/13-1, No. DU 393/9-2, No. ME 5206/3-1, and No. STE 1105/13-1 in the research unit FOR 2440) and the Federal Ministry of Education and Research, Germany (BMBF, Grant No. 05K19WC1) for financial support. D.L. thanks the Alexander von Humboldt Foundation for financial support. N.D. thanks the Swedish Government Strategic Research Area in Materials Science on Functional Materials at Linköping University (Faculty Grant SFO-Mat-LiU No. 2009 00971).

T.M. and F.T. contributed equally to this work.

T.M. and L.D. designed the experiments. T.M., L.D., S.K. and D.L. prepared all diamond anvil cells. G.C., D.L., E.K., T.F., K.G., M.H., M.B., and L.D. performed the diffraction, T.M. and G.C. the NMR experiments. F.T. and G.S.-N. designed and conducted the electronic structure and molecular dynamics calculations. T.M. and F.T. analyzed NMR and XRD data and computational results. T.M., G.S.-N., F.T., L.D., and N.D. assessed all data and wrote the manuscript. 
[1] A. Wurtz, CR Hebd. Seances Acad. Sci. 18, 702 (1844).

[2] R. Mohtadi and S. Orimo, Nat. Rev. Mater. 2, 16091 (2017).

[3] A. P. Drozdov, M. I. Eremets, I. A. Troyan, V. Ksenofontov, and S. I. Shylin, Nature (London) 525, 73 (2015).

[4] M. Somayazulu, M. Ahart, A. K. Mishra, Z. M. Geballe, M. Baldini, Y. Meng, V. V. Struzhkin, and R. J. Hemley, Phys. Rev. Lett. 122, 027001 (2019).

[5] A. P. Drozdov, P. P. Kong, V. S. Minkov, S. P. Besedin, M. A. Kuzovnikov, S. Mozaffari, L. Balicas, F. F. Balakirev, D. E. Graf, V. B. Prakapenka, E. Greenberg, D. A. Knyazev, M. Tkacz, and M. I. Eremets, Nature (London) 569, 528 (2019).

[6] E. Zurek, R. Hoffmann, N. W. Ashcroft, A. R. Oganov, and A. O. Lyakhov, Proc. Natl. Acad. Sci. 106, 17640 (2009).

[7] R. T. Howie, O. Narygina, C. L. Guillaume, S. Evans, and E. Gregoryanz, Phys. Rev. B 86, 064108 (2012).

[8] S. Aldridge and A. J. Downs, Chem. Rev. 101, 3305 (2001).

[9] R. Burtovyy and M. Tkacz, Solid State Commun. 131, 169 (2004).

[10] C. Donnerer, T. Scheler, and E. Gregoryanz, J. Chem. Phys. 138, 134507 (2013).

[11] J. Binns, M. Peña-Alvarez, M.-E. Donnelly, E. Gregoryanz, R. T. Howie, and P. Dalladay-Simpson, Engineering 5, 505 (2019).

[12] N. Spengler, J. Höfflin, A. Moazenzadeh, D. Mager, N. MacKinnon, V. Badilita, U. Wallrabe, and J. G. Korvink, PLoS ONE 11, e0146384 (2016).

[13] M. Jouda, R. Kamberger, J. Leupold, N. Spengler, J. Hennig, O. Gruschke, and J. G. Korvink, Concepts Magn. Reson. Part B Magn. Reson. Eng. 47B, e21357 (2017).

[14] T. Meier, N. Wang, D. Mager, J. G. Korvink, S. Petitgirard, and L. Dubrovinsky, Sci. Adv. 3, eaao5242 (2017).

[15] T. Meier, Prog. Nucl. Magn. Reson. Spectrosc. 106-107, 26 (2018).

[16] L. J. Farrugia, J. Appl. Crystallogr. 32, 837 (1999).

[17] T. Meier, in Annual Reports on NMR Spectroscopy, edited by G. Webb, 93rd ed. (Elsevier, London, 2018), pp. 1-74.

[18] T. Meier, F. Trybel, S. Khandarkhaeva, G. Steinle-Neumann, S. Chariton, T. Fedotenko, S. Petitgirard, M. Hanfland, K. Glazyrin, N. Dubrovinskaia, and L. Dubrovinsky, Phys. Rev. X 9, 031008 (2019).

[19] T. Meier, S. Petitgirard, S. Khandarkhaeva, and L. Dubrovinsky, Nat. Commun. 9, 2766 (2018).

[20] O. Narygina, L. S. Dubrovinsky, C. A. McCammon, A. Kurnosov, I. Y. Kantor, V. B. Prakapenka, and N. A. Dubrovinskaia, Earth Planet. Sci. Lett. 307, 409 (2011).

[21] N. Dubrovinskaia, L. Dubrovinsky, R. Caracas, and M. Hanfland, Appl. Phys. Lett. 97, 251903 (2010).

[22] C. Prescher and V. B. Prakapenka, High Press. Res. 35, 223 (2015).

[23] V. Petrícek, M. Dušek, and L. Palatinus, Z. Krist. 229, 345 (2014).

[24] G. M. Sheldrick, Acta Crystallogr. Sect. A 64, 112 (2007).

[25] G. M. Sheldrick, Acta Crystallogr. 71, 3 (2015).

[26] H. Geen and R. Freeman, J. Magn. Reson. 93, 93 (1991).

[27] See Supplemental Material at http://link.aps.org/supplemental/ 10.1103/PhysRevB.102.165109 for experimental and computational details and additional figures.

[28] N. Bloembergen and T. J. J. Rowland, Acta Metall. 1, 731 (1953).

[29] G. E. Pake, J. Chem. Phys. 16, 327 (1948).
[30] C. E. Hughes and K. D. M. Harris, Solid State Nucl. Magn. Reson. 80, 7 (2016).

[31] R. K. Harris, E. D. Becker, S. M. Cabral de Menezes, P. Granger, R. E. Hoffman, and K. W. Zilm, Pure Appl. Chem. 80, 59 (2008).

[32] P. Giannozzi, S. Baroni, N. Bonini, M. Calandra, R. Car, C. Cavazzoni, D. Ceresoli, G. L. Chiarotti, M. Cococcioni, I. Dabo, A. Dal Corso, S. de Gironcoli, S. Fabris, G. Fratesi, R. Gebauer, U. Gerstmann, C. Gougoussis, A. Kokalj, M. Lazzeri, L. Martin-Samos, N. Marzari, F. Mauri, R. Mazzarello, S. Paolini, A. Pasquarello, L. Paulatto, C. Sbraccia, S. Scandolo, G. Sclauzero, A. P. Seitsonen, A. Smogunov, P. Umari, and R. M. Wentzcovitch, J. Phys.: Condens. Matter 21, 395502 (2009).

[33] P. Giannozzi, O. Andreussi, T. Brumme, O. Bunau, M. Buongiorno Nardelli, M. Calandra, R. Car, C. Cavazzoni, D. Ceresoli, M. Cococcioni, N. Colonna, I. Carnimeo, A. Dal Corso, S. de Gironcoli, P. Delugas, R. A. DiStasio, A. Ferretti, A. Floris, G. Fratesi, G. Fugallo, R. Gebauer, U. Gerstmann, F. Giustino, T. Gorni, J. Jia, M. Kawamura, H.-Y. Ko, A. Kokalj, E. Küçükbenli, M. Lazzeri, M. Marsili, N. Marzari, F. Mauri, N. L. Nguyen, H.-V. Nguyen, A. Otero-de-la-Roza, L. Paulatto, S. Poncé, D. Rocca, R. Sabatini, B. Santra, M. Schlipf, A. P. Seitsonen, A. Smogunov, I. Timrov, T. Thonhauser, P. Umari, N. Vast, X. Wu, and S. Baroni, J. Phys.: Condens. Matter 29, 465901 (2017).

[34] P. E. Blöchl, Phys. Rev. B 50, 17953 (1994).

[35] J. P. Perdew, K. Burke, and M. Ernzerhof, Phys. Rev. Lett. 77, 3865 (1996).

[36] T. Sjostrom and S. Crockett, Phys. Rev. E 97, 053209 (2018).

[37] H. J. Monkhorst and J. D. Pack, Phys. Rev. B 13, 5188 (1976).

[38] R. E. Cohen, O. Gülseren, and R. J. Hemley, Am. Mineral. 85, 338 (2000).

[39] H. C. Andersen, J. Chem. Phys. 72, 2384 (1980).

[40] X. W. Zhou, T. W. Heo, B. C. Wood, V. Stavila, S. Kang, and M. D. Allendorf, J. Appl. Phys. 123, 225105 (2018).

[41] A. Dewaele, P. Loubeyre, and M. Mezouar, Phys. Rev. B 70, 094112 (2004).

[42] M. Tkacz, S. Majchrzak, and B. Baranowski, Z. Phys. Chem. 163, 467 (1989).

[43] R. Goring, R. Lukas, and K. Bohmhammel, J. Phys. C 14, 5675 (1981).

[44] D. S. Schreiber and R. M. Cotts, Phys. Rev. 131, 1118 (1963).

[45] D. S. Schreiber and L. D. Graham, J. Chem. Phys. 43, 2573 (1965).

[46] S. Kazama and Y. Fukai, J. Phys. Soc. Japan 42, 119 (1977).

[47] S. Kazama and Y. Fukai, J. Less-Common Met. 53, 25 (1977).

[48] X. H. Xiao, D. F. Duan, Y. Bin Ma, H. Xie, H. Song, D. Li, F. B. Tian, B. B. Liu, H. Y. Yu, and T. Cui, Front. Phys. 14, 2 (2019).

[49] W. S. Price, Concepts Magn. Reson. Part A 34A, 60 (2009).

[50] A. Abragam and L. C. Hebel, Principles of Nuclear Magnetism, 18th ed. (Oxford University Press, Oxford New York, 1961).

[51] J. Korringa, Physica 16, 601 (1950).

[52] W. Heitler and E. Teller, Proc. R. Soc. London Ser. A 155, 629 (1936).

[53] F. Kimmerle, G. Majer, U. Kaess, A. J. Maeland, M. S. Conradi, and A. F. McDowell, J. Alloys Compd. 264, 63 (1998).

[54] N. Bloembergen, E. M. Purcell, and R. V Pound, Phys. Rev. 73, 679 (1948).

[55] R. C. Bowman, A. Attalla, and W. E. Tadlock, Int. J. Hydrogen Energy 1, 421 (1977). 
[56] A. F. McDowell, C. F. Mendelsohn, M. S. Conradi, R. C. Bowman, and A. J. Maeland, Phys. Rev. B 51, 6336 (1995).

[57] R. C. Bowman, D. M. Gruen, and M. H. Mendelsohn, Solid State Commun. 32, 501 (1979).

[58] G. Majer, W. Renz, A. Seeger, R. G. Barnes, J. Shinar, and A. V. Skripov, J. Alloys Compd. 231, 220 (1995).

[59] H. Wipf, B. Kappesser, and R. Werner, J. Alloys Compd. 310, 190 (2000).

[60] F. Peng, Y. Sun, C. J. Pickard, R. J. Needs, Q. Wu, and Y. Ma, Phys. Rev. Lett. 119, 107001 (2017).
[61] Z. M. Geballe, H. Liu, A. K. Mishra, M. Ahart, M. Somayazulu, Y. Meng, M. Baldini, and R. J. Hemley, Angew. Chemie - Int. Ed. 57, 688 (2018).

[62] E. Zurek and T. Bi, J. Chem. Phys. 150, 050901 (2019).

[63] H. Liu, I. I. Naumov, Z. M. Geballe, M. Somayazulu, J. S. Tse, and R. J. Hemley, Phys. Rev. B 98, 100102(R) (2018).

[64] I. Errea, F. Belli, L. Monacelli, A. Sanna, T. Koretsune, T. Tadano, R. Bianco, M. Calandra, R. Arita, F. Mauri, and J. A. Flores-Livas, Nature (London) 578, 66 (2020). 